TITLE:

\title{
Alkali-metal adsorption and manipulation on a hydroxylated TiO2(110) surface using atomic force microscopy
}

\section{AUTHOR(S):}

Yurtsever, Ayhan; Sugimoto, Yoshiaki; Abe, Masayuki; Matsunaga, Katsuyuki; Tanaka, Isao; Morita, Seizo

\section{CITATION:}

Yurtsever, Ayhan ...[et al]. Alkali-metal adsorption and manipulation on a hydroxylated TiO2(110) surface using atomic force microscopy. PHYSICAL REVIEW B 2011, 84(8): 085413.

\section{ISSUE DATE:}

2011-08

URL:

http://hdl.handle.net/2433/161784

\section{RIGHT:}

(c)2011 American Physical Society 
PHYSICAL REVIEW B 84, 085413 (2011)

\title{
Alkali-metal adsorption and manipulation on a hydroxylated $\mathrm{TiO}_{2}(110)$ surface using atomic force microscopy
}

\author{
Ayhan Yurtsever, ${ }^{1, *}$ Yoshiaki Sugimoto, ${ }^{1}$ Masayuki Abe, ${ }^{1}$ Katsuyuki Matsunaga,${ }^{2,3}$ Isao Tanaka, ${ }^{2}$ and Seizo Morita ${ }^{1}$ \\ ${ }^{1}$ Graduate School of Engineering, Osaka University 2-1 Yamada-Oka, Suita, Osaka 565-0871, Japan \\ ${ }^{2}$ Graduate School of Engineering, Kyoto University, Yoshida, Sakyo, Kyoto 606-8501, Japan \\ ${ }^{3}$ Graduate School of Engineering, Nagoya University, Furo-cho, Chikusa, Nagoya 464-8603, Japan \\ (Received 21 March 2011; revised manuscript received 14 July 2011; published 22 August 2011)
}

\begin{abstract}
We report on the adsorption and manipulation of $\mathrm{K}$ atoms on a hydroxylated $\mathrm{TiO}_{2}(110)-1 \times 1$ surface using low-temperature noncontact atomic force microscopy. At low coverage, first-principles GGA $+U$ calculations reveal favorable adsorption sites that are consistent with the experimentally observed adsorption positions on the upper-threefold hollow site, in which the $\mathrm{K}$ atom is bound to two bridging-oxygen $\left(\mathrm{O}_{\mathrm{br}}\right)$ and one in-plane oxygen atoms, forming an ionic bond with three oxygen by transferring almost one of the $\mathrm{K} 4 \mathrm{~s}$ electrons to the substrate. $\mathrm{K}$ atoms can be manipulated preferentially along the [001] direction over the $\mathrm{O}_{\mathrm{br}}$ row by the tip through attractive force. Density functional theory calculations have provided diffusion paths for the adsorbed $\mathrm{K}$ atoms, which indicate a channel that provides ease of diffusion for a single $\mathrm{K}$ atom along the $\mathrm{O}_{\mathrm{br}}$ rows associated with an energy barrier of $0.21 \mathrm{eV}$.
\end{abstract}

DOI: 10.1103/PhysRevB.84.085413

\section{INTRODUCTION}

Among the metal oxides used as supports, rutile $\mathrm{TiO}_{2}(110)$ has been regarded as a model system for other metal-oxide surface studies and is one of the most intensively studied systems in surface science, due to its various applications, including heterogeneous catalysis, solar cells, photocatalytic water splitting, protective coatings, and gas-sensing devices. ${ }^{1,2}$ Addition of alkali-metal (AM) atoms to $\mathrm{TiO}_{2}$ surfaces significantly modifies the surface electronic properties, inducing a reduction in the local work function. ${ }^{3-5}$ The interaction of AMs with metal oxide supports plays a crucial role in catalysis as a reaction promoter. ${ }^{1}$ It has been also demonstrated that the catalytic activity and selectivity of metal-oxide catalysts dispersed on $\mathrm{TiO}_{2}(110)$ is significantly dependent on the size and distribution of the metal clusters, ${ }^{6}$ and only a particular size of nanoclusters exhibits high catalytic activity. Therefore, control of the structural properties of alkali-metal clusters is very important for improving catalytic performance. $\mathrm{K} / \mathrm{TiO}_{2}(110)$ was selected as a model system for AM atommanipulation experiments, because the adsorption of $\mathrm{K}$ on the $\mathrm{TiO}_{2}(110)$ surface has been studied extensively using various spectroscopic techniques. ${ }^{3,7}$ It is well established that the charges are transferred from $\mathrm{K}$ to the substrate at low $\mathrm{K}$ coverage. Additionally, the catalytic properties of $\mathrm{K}$ and its coadsorption with Au have received much attention, both experimentally and theoretically, ${ }^{8,9}$ and its significant effect on the binding sites and cluster sizes of Au nanoparticles has been demonstrated.

Noncontact atomic force microscopy (nc-AFM) has proven to be a powerful and convenient tool for the formation of ordered nanostructures on various surfaces, such as semiconductors, ${ }^{10,11}$ metals, ${ }^{12}$ and insulators. ${ }^{13,14}$ Despite the importance of adsorbate atom manipulation on the $\mathrm{TiO}_{2}(110)$ surface to tailor the catalytic efficiency for heterogeneous catalysis, very little is known about the possible pathways of metal adsorbates and their interaction with the $\mathrm{TiO}_{2}(110)$ surface. Therefore, nc-AFM experimentation combined with first-principles density functional theory (DFT) calculations was considered a viable approach to obtain an atomic-scale understanding of the chemical adsorption sites and diffusion
PACS number(s): 81.16.Ta, 68.37.Ps, 68.43.Fg, 68.47.Gh

paths of AM atoms dispersed on metal-oxide surfaces such as $\mathrm{TiO}_{2}(110)$.

Herein, we present an investigation of the adsorption and tip-induced motion of $\mathrm{K}$ atoms on a hydroxylated $\mathrm{TiO}_{2}(110)$ surface by combining low-temperature nc-AFM experiments and first-principles DFT calculations. For a single $\mathrm{K}$ atom, we determine and discuss the possible adsorption sites. The diffusion barriers for a single $\mathrm{K}$ atom-associated with different pathways-were determined, which can provide valuable information on the possible channels for AM atom manipulation on oxide surfaces.

\section{EXPERIMENTAL PROCEDURES}

Experiments were carried out using a home-built ultrahighvacuum nc-AFM ${ }^{15}$ operated at low temperature $(80 \mathrm{~K})$ with a base pressure better than $5 \times 10^{-11}$ Torr. The nc-AFM was operated using the frequency modulation detection mode, ${ }^{16}$ keeping the cantilever oscillation amplitude constant. Prior to use for imaging, commercial $\mathrm{Si}$ tips were cleaned via Ar-ion sputtering to remove the native oxide layer and other contaminants. The clean rutile $\mathrm{TiO}_{2}(110)$ crystal (Shinko) used in these experiments was prepared using repeated cycles of $\mathrm{Ar}^{+}$ion bombardment $\left(2 \mathrm{keV}, 3 \times 10^{-6}\right.$ Torr $)$ for approximately $5 \mathrm{~min}$ at room temperature (RT) and annealing to approximately $1000 \mathrm{~K}$ for $1 \mathrm{~min}$ to restore a flat substrate surface. The $\mathrm{OH}$ groups on the $\mathrm{TiO}_{2}(110)$ surface were spontaneously created from the dissociation of water molecules (from background residual gas) over the oxygen vacancy sites by transferring the $\mathrm{H}$ atom to a neighboring $\mathrm{O}_{\mathrm{br}}$ sites. ${ }^{17}$ By keeping the freshly prepared sample in UHV more than $3 \mathrm{~h}$ at RT before observing at $80 \mathrm{~K}$, all of the $\mathrm{O}$ vacancies are occupied by OHs. Thus, in our measurements, the $\mathrm{OH}$ groups are considered to be the most common surface impurity defects.

$\mathrm{K}$ atoms were deposited onto the $\mathrm{TiO}_{2}$ surface from a SAES Getter source (previously outgassed) heated with $6.5 \mathrm{~A}$ of current, with the pressure kept at $\leqslant 3 \times 10^{-10}$ Torr during the deposition. A K coverage surface was obtained after a $20 \mathrm{~s}$ 
deposition onto the sample held at RT located $10 \mathrm{~cm}$ apart from the source. After exposing the surface to $\mathrm{K}$ atoms, the sample was immediately transferred into the observation chamber that houses the nc-AFM unit at $80 \mathrm{~K}$. The complete adsorption and transfer process took approximately $2 \mathrm{~min}$. In order to minimize the long-range electrostatic force during topographic imaging, the average contact potential difference (CPD) was compensated by application of a sample bias in the range of $0.5-1.5 \mathrm{~V}$ with the tip being held at ground potential. For all images shown here, the fast scan direction is from left to right.

\section{COMPUTATIONAL METHOD}

First-principles DFT calculations using a spin-polarized generalized-gradient approximation, including on-site Coulomb repulsion correction ${ }^{18}$ and projector-augmented wave potentials as implemented in the VASP code, ${ }^{19,20}$ were performed to assist in the determination of the most favorable adsorption sites for $\mathrm{K}$ on rutile $\mathrm{TiO}_{2}(110)$ surfaces. Electronic wave functions were expanded by plane waves up to an energy cutoff of $500 \mathrm{eV}$. An effective $U$ parameter of $5.8 \mathrm{eV}$ was applied to Ti-3d electrons. This $U$ value was previously reported to appropriately describe defects in $\mathrm{TiO}_{2}$ systems. ${ }^{21}$

Potassium adsorption on the $\mathrm{TiO}_{2}$ surface was simulated by periodic supercells involving the (110) surface slab. K atoms were adsorbed on the (110) surfaces at both ends of the slab. To prevent spurious interaction between $\mathrm{K}$ atoms on the surfaces, the slab was set to have a thickness of about $22 \AA$ and was sandwiched by vacuum layers with a thickness of more than $10 \AA$. Differently sized slabs along the $\langle 001\rangle$ direction on the surface plane were also used $(1 \times 1$ and $1 \times 2$ surface unit) to check an effect of density of adsorbed $\mathrm{K}$ atoms. The resultant total numbers of atoms in the supercells were 44 for the $1 \times 1$ and 86 for the $1 \times 2$ surface-unit model, respectively. Atomic positions within the supercells were allowed to relax until their forces became less than $0.01 \mathrm{eV} / \AA$. Brillouinzone sampling for the electronic structure calculations was performed using a $4 \times 2 \times 1$ Monkhorst-Pack (MP) mesh $^{22}$ for the $1 \times 1$ model and a $2 \times 2 \times 1$ MP mesh for the $1 \times 2$ model. Test calculations using denser MP meshes for some kinds of $\mathrm{K}$ adsorption sites ensure a good accuracy of the present calculations within an error of about $0.01 \mathrm{eV}$ in adsorption energy. Here the adsorption energies of potassium on the surface were evaluated from the total energy of the $\mathrm{K}$-adsorbed surface minus those of the pristine $\mathrm{TiO}_{2}$ surface and the isolated $\mathrm{K}$ atom. Therefore, more positive adsorption energies mean stronger attractive interactions between $\mathrm{K}$ and the surface.

The nudged elastic band (NEB) method ${ }^{23}$ was applied to analyze the diffusive motion of adsorbed $\mathrm{K}$ atom observed by the present nc-AFM technique. The NEB method is an effective way to find minimum energy pathway (MEP) of a diffusive species between an initial and a final state, both of which are at local minima on potential energy surface. A string of images (replicas) is prepared along the pathway, and the images are connected by spring forces, mimicking an elastic band. In this study, the images were then relaxed until forces perpendicular to and along the band converge below a particular criteria (in this case, $0.03 \mathrm{eV} / \AA$ ) .

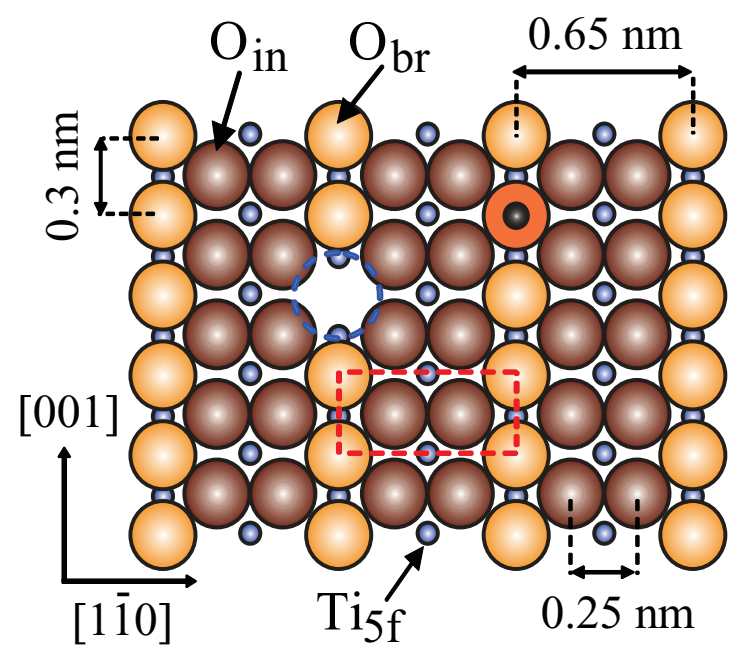

FIG. 1. (Color online) Two dimensional ball model of rutile $\mathrm{TiO}_{2}(110)-(1 \times 1)$ showing the structure of the surface. The surface consists of rows of $\mathrm{O}_{\mathrm{br}}$ atoms (yellow spheres) protruding above the surface, and rows of fivefold coordinated $\mathrm{Ti}$ atoms $\left(\mathrm{Ti}_{5 \mathrm{f}}\right)$ surrounded by in-plane oxygen atoms. The oxygen vacancy (marked with a blue dashed-circle) and $\mathrm{OH}$ group (orange sphere with a smaller black sphere on it) are also indicated in the model. The red (dark gray) dashed-rectangle shows the $\mathrm{TiO}_{2}(110)$ surface unit cell.

\section{RESULTS AND DISCUSSION}

\section{A. Adsorption sites for a single $\mathbf{K}$ atom}

Atomic-scale structures of pristine $\mathrm{TiO}_{2}(110)-1 \times 1$ surfaces have been resolved both by scanning tunneling microscopy $(\mathrm{STM})^{24}$ and by nc- $\mathrm{AFM}^{25}$ and consist of alternating rows of fivefold coordinated $\mathrm{Ti}^{4+}\left(\mathrm{Ti}_{5 f}\right)$ and twofold coordinated bridging $\mathrm{O}^{2-}\left(\mathrm{O}_{\mathrm{br}}\right)$ atoms aligned along the [001] direction (see Fig. 1). Contrary to the STM imaging contrast of the $\mathrm{TiO}_{2}$ surface that relies on electronic rather than geometric effects (with $\mathrm{Ti}_{5 f}$ atoms as protruding rows), three different contrast modes have been routinely obtained for $\mathrm{TiO}_{2}(110)$ surfaces in nc-AFM, which are classified ${ }^{26}$ as neutral (imaging of $\mathrm{O}_{\mathrm{br}}$ sites together with $\mathrm{OH}$ groups as protrusions), hole (imaging of $\mathrm{O}_{\mathrm{br}}$ rows as bright stripes and $\mathrm{OH}$ groups as dark hole), and protrusion modes (imaging of $\mathrm{Ti}_{5 f}$ atoms and $\mathrm{OH}$ groups as protrusions).

Figure 2(a) shows an nc-AFM image of a bare $\mathrm{TiO}_{2}(110)$ surface prior to $\mathrm{K}$-atom exposure, with $\mathrm{OH}$ groups in the $\mathrm{O}_{\mathrm{br}}$ rows obtained with a neutrally terminated tip, which provides a contrast resembling the true geometric structure of the substrate. A typical nc-AFM topography image of the $\mathrm{K}$ atoms at $80 \mathrm{~K}$, obtained after exposure of the hydroxylated $\mathrm{TiO}_{2}(110)$ surface to $\mathrm{K}$, is shown in Fig. 2(c); this has been referred to as the protrusion mode. ${ }^{26}$ The $\mathrm{Ti}_{5 f}$ atomic rows are imaged as bright stripes (strong attractive force), while the dark troughs (weak attractive force) correspond to the positions of $\mathrm{O}_{\mathrm{br}}$ atoms. The small, bright features homogeneously distributed over the $\mathrm{O}_{\mathrm{br}}$ atomic rows have been identified as single $\mathrm{OH}$ impurity defects.

At low $\mathrm{K}$ coverage, the $\mathrm{K}$ atoms, which appear as brighter protrusions relative to the $\mathrm{OH}$ and $\mathrm{Ti}_{5 f}$ atoms, preferentially adsorb at the edges of the $\mathrm{O}_{\mathrm{br}}$ rows, as indicated by the dashed stripes in Fig. 2(d). Figure 2(b) shows the hidden 

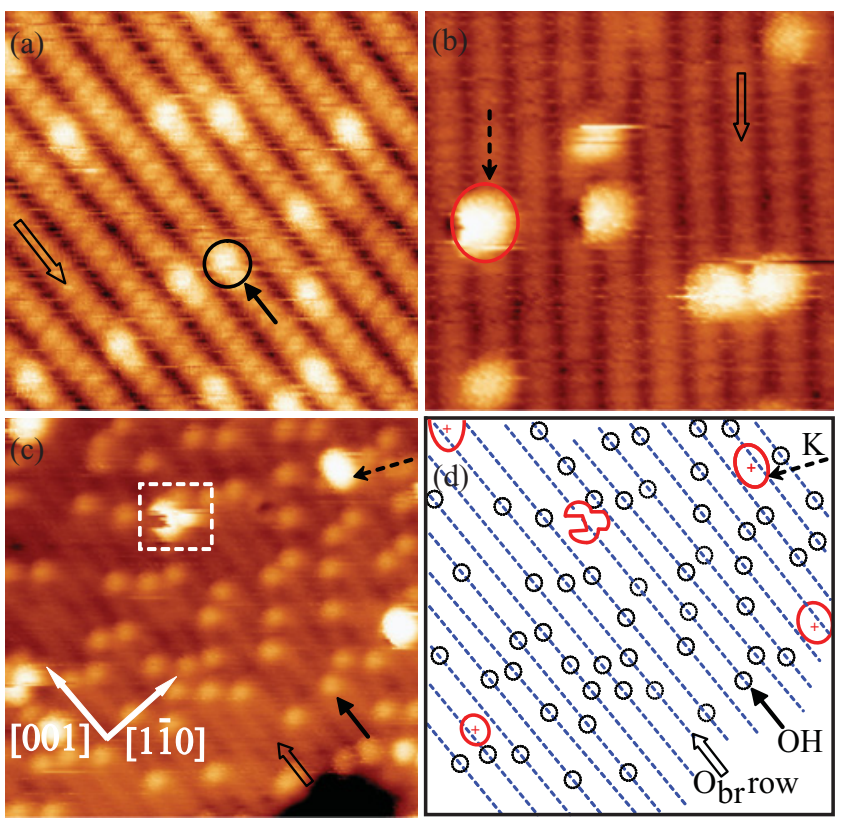

FIG. 2. (Color online) nc-AFM topography images of a hydroxylated $\mathrm{TiO}_{2}(110)$ surface at $80 \mathrm{~K}$. (a) Prior to K-atom deposition $\left(6.8 \times 5.6 \mathrm{~nm}^{2}\right)$ and (b) after exposure to $\mathrm{K}$ atom $\left(6.8 \times 6.8 \mathrm{~nm}^{2}\right)$, which has been referred to as hidden mode image contrast of the surface. (c) Protrusion mode image of the surface $\left(12 \times 12 \mathrm{~nm}^{2}\right)$ after exposure to $\mathrm{K}$ atoms. The dashed rectangle indicates the switching of K-atom position. (d) Schematic model of the surface shown in (c), where black open circles represent $\mathrm{OH}$ groups (marked by black arrows), red (dark gray) ellipses indicate $\mathrm{K}$ atoms (marked by dashed black arrows), and dashed blue lines superimposed onto the $\mathrm{O}_{\mathrm{br}}$ atomic rows (marked by open thick black arrows) passing through the $\mathrm{OH}$ groups illustrate that the $\mathrm{K}$ atoms are not predominantly located on the center of the $\mathrm{Ti}_{5 f}$ rows but are slightly shifted toward the $\mathrm{O}_{\mathrm{br}}$ rows. The acquisition parameters were (a) $f_{0}=165.191 \mathrm{kHz}$, $k=52.7 \mathrm{~N} / \mathrm{m}, \Delta f=-5.3 \mathrm{~Hz}$, and $A=13.4 \mathrm{~nm}$; (b) $f_{0}=165.188$ $\mathrm{kHz}, k=52.7 \mathrm{~N} / \mathrm{m}, \Delta f=-4.5 \mathrm{~Hz}$, and $A=10.3 \mathrm{~nm}$; (c) $f_{0}=$ $165.186 \mathrm{kHz}, k=52.7 \mathrm{~N} / \mathrm{m}, \Delta f=-4.5 \mathrm{~Hz}$, and $A=12.5 \mathrm{~nm}$. The slow scan direction is from top to bottom.

mode nc-AFM image of $\mathrm{K}$ adsorbed on the $\mathrm{TiO}_{2}(110)$ surface, where no $\mathrm{OH}$ impurity defects were observed, which would usually appear in common nc-AFM images obtained under similar sample preparation conditions. Based on our previous nc-AFM study, ${ }^{27}$ we have identified the protruding atomic rows obtained in the "hidden mode" image contrast as $\mathrm{O}_{\mathrm{br}}$ atoms. It is particular noteworthy that the $\mathrm{K}$ atoms appear as a bright protrusion in these two distinctly different image contrast modes even when the chemical state of tip-apex is entirely changed. Similarly to the previous case and as expected, the $\mathrm{K}$-atom protrusions are located neither on top of $\mathrm{O}_{\mathrm{br}}$ rows nor in the center of the troughs (i.e., $\mathrm{Ti}_{5 f}$ atomic rows) but appear to be shifted slightly toward one of the $\mathrm{O}_{\mathrm{br}}$ rows. Therefore, we tentatively conclude that the $\mathrm{K}$ atoms are bound either on two $\mathrm{O}_{\mathrm{br}}$ and one in-plane oxygen $\left(\mathrm{O}_{\text {in }}\right)$ atoms or two $\mathrm{O}_{\text {in }}$ and one $\mathrm{O}_{\mathrm{br}}$ atoms on the triangular plane of the surface; a so-called hollow site. However, precise assignment of the $\mathrm{K}$ adsorption site cannot be made by only experiment.

In order to clarify adsorption sites of a potassium atom on the $\mathrm{TiO}_{2}(110)$ surfaces theoretically, first-principles DFT
TABLE I. Adsorption energies and optimized bond lengths for the $\mathrm{K}$ atom adsorbed on various sites of the $\mathrm{TiO}_{2}$ surface, obtained using GGA $+U$ calculations. The values in parentheses show the results of the calculations for cell $1 \times 1$.

\begin{tabular}{lcccc}
\hline \hline Sites & $E_{b}(\mathrm{eV})$ & $\mathrm{K}-\mathrm{O}_{\text {br }}(\AA)$ & $\mathrm{K}^{-} \mathrm{O}_{\text {in }}(\AA)$ & $\mathrm{K}^{-\mathrm{Ti}_{5 f}}(\AA)$ \\
\hline 3 & $2.70(1.41)$ & 2.52 & 2.70 & - \\
6 & $2.60(1.36)$ & 2.41 & 2.80 & - \\
4 & $2.54(1.26)$ & 2.43 & - & - \\
1 & $2.13(0.92)$ & - & - & 2.91 \\
5 & $2.01(0.90)$ & 2.23 & - & - \\
2 & $1.66(0.93)$ & - & 2.67 & 3.31 \\
\hline \hline
\end{tabular}

calculations were performed. Plausible binding sites and corresponding binding energies were determined by employing two different cells, $(1 \times 2)$ and $(1 \times 1)$, the details of which are summarized in Table I. Three possible stable sites were obtained for $\mathrm{K}$ atoms on a $\mathrm{TiO}_{2}(110)$ surface at low coverage (see Table I). The threefold hollow sites [sites 3 and 6 in Fig. 6(b)] and the bridge position between two $\mathrm{O}_{\mathrm{br}}$ atoms (site 4) have calculated adsorption energies of 2.70, 2.60, and $2.54 \mathrm{eV}$, respectively. In accordance with nc-AFM experiments, DFT calculations predict that the most stable binding site for a single $\mathrm{K}$ atom is the upper-threefold hollow site (site 3 ) surrounded by two $\mathrm{O}_{\mathrm{br}}$ and one $\mathrm{O}_{\text {in }}$ atoms with $\mathrm{K}-\mathrm{O}_{\text {br }}$ and $\mathrm{K}-\mathrm{O}_{\text {in }}$ bond lengths of 2.52 and $2.70 \AA$, respectively, which is in excellent agreement with the values of $2.49 \pm 0.12$ and $2.73 \pm 0.07 \AA$ obtained from surface extended $\mathrm{x}$-ray-absorption fine-structure (SEXAFS) spectra. ${ }^{28}$ Besides the individual $\mathrm{K}$ atoms on the surface, some atomic species appear to reside on the surface $\mathrm{Ti}_{5 f}$ rows in some nc-AFM images, which cannot be assigned unambiguously and not set in motion even by the influence of the tip. These species most likely originate from the impurities or $\mathrm{K}$ atoms that interact with surface $\mathrm{Ti}_{5 f}$ defect sites. The DFT calculations cannot determine the stable sites for $\mathrm{K}$ atoms on the $\mathrm{Ti}_{5 f}$ atomic rows. Adsorption on the $\mathrm{Ti}_{5 f}$ row at the surface is approximately $0.57 \mathrm{eV}$ less favorable than adsorption on a hollow site.

As summarized in Table I, the configuration corresponding to site 3 is predicted to be approximately $0.1 \mathrm{eV}$ more favorable than $\mathrm{K}$ adsorbed on the lower-hollow site (site 6). The computed energy difference between hollow sites can be rationalized in terms of the local chemical environment; the upper hollow site is more electronegative than the lower hollow site (i.e., site 6), due to the higher coordination number of $\mathrm{O}_{\text {in }}$ atoms. Therefore, at this adsorption site, stronger ionic bonds can be formed with more electronegative $\mathrm{O}_{\mathrm{br}}$ atoms. These results are further supported by the Bader charge analysis of the $\mathrm{K}$ adsorbed surface at site 3 , which reveals that the nature of $\mathrm{K}$ bonding on the surface originates from ionic interaction with a large charge transfer of approximately $0.85 e$ from the $\mathrm{K} 4 s$ state to the $\mathrm{TiO}_{2}(110)$ surface layer, and this results in a higher oxidation state. As shown in Fig. 3, the analysis of the density of states (DOS) at site 3 shows that the extra electronic level occupied by one electron is present just above the upper valence band, upon the $\mathrm{K}$-atom adsorption. The wave function is found to be composed of Ti $3 d$ and $\mathrm{O} 2 p$ orbitals in the Ti-O layers below the surface. It can be considered, therefore, that the electron from the adsorped $\mathrm{K}$ atom goes 


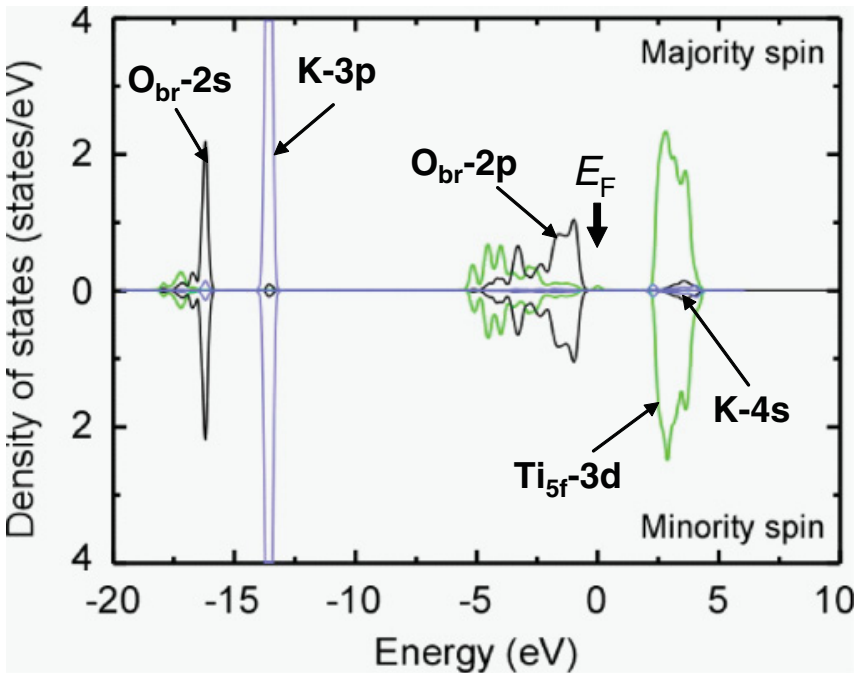

FIG. 3. (Color online) Majority and minority spin components of the local density of states (LDOS) of K (adsorbed at upper hollow site 3 ) and surface ions on the $\mathrm{TiO}_{2}(110)$ surface. The green and black curves represent the LDOS of $\mathrm{Ti}_{5 f}$ and $\mathrm{O}_{\mathrm{br}}$ atoms, respectively. The blue line indicates the LDOS of $\mathrm{K}$ atom.

below the $\mathrm{TiO}_{2}(110)$ surface layer. The charge transfer was also confirmed by Kelvin probe force microscopy (KPFM) images of the $\mathrm{K} / \mathrm{TiO}_{2}$ surface, which indicate a reduction in the local contact potential difference (LCPD) of the K atoms with respect to the supporting $\mathrm{TiO}_{2}$ surface. This reduction in the LCPD signal was attributed to the formation of the electrical dipole resulting from the $\mathrm{K}-\mathrm{TiO}_{2}$ charge transfer. The details of the KPFM results will be discussed elsewhere. ${ }^{29}$

\section{B. Manipulation of $\mathrm{K}$ atom on hydroxylated $\mathrm{TiO}_{2}$ surface}

In addition to stable imaging of $\mathrm{K}$ atoms on the surface at $80 \mathrm{~K}$, adsorbed $\mathrm{K}$ atoms become mobile when the tip-sample distance is reduced (stronger tip-adsorbate interaction). The displacement of a single $\mathrm{K}$ atom can be initiated along the easiest available paths over the surface by adjusting the tip-sample distances. An example of tip-induced K-atom manipulation-achieved with a tip producing protrusion mode contrast of the surface-is demonstrated in Fig. 4(a). Under the influence of the tip, single $\mathrm{K}$ atoms appear as a bright line atomic feature in the resulting nc-AFM image, which manifests the manipulation events along the [001] direction. Closer inspection of this bright line in Fig. 4(c) reveals that the apparent atomic line is the result of single $\mathrm{K}$-atom movement on the $\mathrm{O}_{\mathrm{br}}$ row by discrete hopping from one equilibrium surface site to another with a periodicity of the underlying $\mathrm{O}_{\mathrm{br}}$ atoms of approximately $2.9 \AA$. The trajectory of the moving K atoms is centered over the $\mathrm{O}_{\mathrm{br}}$ row and is extended over two $\mathrm{Ti}_{5 f}$ atomic rows along the [110] direction.

This type of atom displacement was previously observed in the STM manipulation of $\mathrm{Ag}$ atoms on the $\mathrm{Ag}(110)$ surface. ${ }^{30}$ Comparison of the shape (i.e., a discrete convex half-moon) of the K atoms under tip-induced motion [see Figs. 4(a) and 4(c)] with the previously reported results from STM manipulation experiments conducted at cryogenic temperatures reveals that the manipulation mode in this experiment can be classified as pulling mode, ${ }^{30,31}$ where the adsorbate is pulled by an attractive

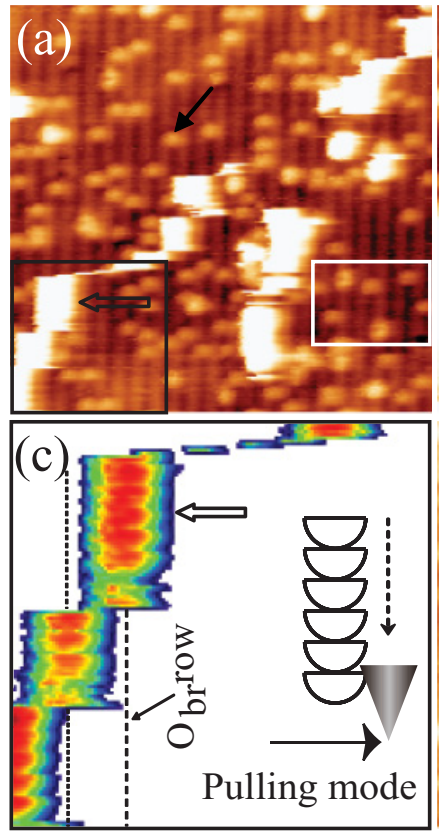

(e) (1)

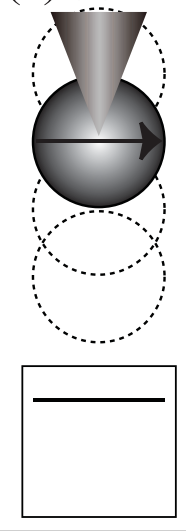

(2)

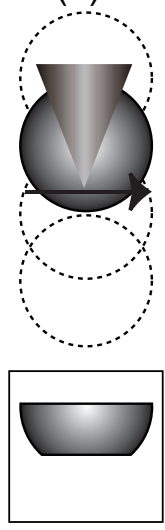

(3)

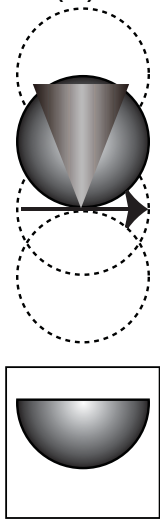

(4)

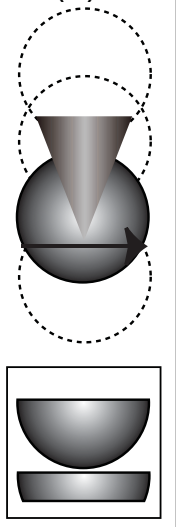

FIG. 4. (Color online) nc-AFM images of the $\mathrm{TiO}_{2}(110)$ surface $\left(16 \times 16 \mathrm{~nm}^{2}\right)$, which illustrate the mobility of $\mathrm{K}$ atoms observed with a tip-generating protrusion mode image contrast of the surface at $80 \mathrm{~K}$. Images (a), (b), and (d) were taken almost in the same region of the surface area. Image (d) was obtained with a larger tip-sample distance. White rectangles were used to mark the same position. The $\mathrm{K}$ atoms preferentially migrate along the $\mathrm{O}_{\mathrm{br}}$ row. The black and open thick black arrows indicate $\mathrm{OH}$ groups and $\mathrm{K}$ atoms, respectively. (c) Zoom into the marked area in (a), revealing the distinctive motion of $\mathrm{K}$ atoms that resemble the shape of a half-moon. (e) Schematic illustrations to explain the mechanism of the tip-induced pulling mode manipulation of K atoms. The lower panel of (e) shows the appearance of $\mathrm{K}$ atom with respect to tip scan position. The acquisition parameters were [(a), (b), and (d)] $f_{0}=165.189 \mathrm{kHz}, k=52.7 \mathrm{~N} / \mathrm{m}, U_{\text {cpd }}=1.0$ $\mathrm{V}$, and $A=15.7 \mathrm{~nm}$. Imaging set point for (a) and (b) $\Delta f=-4.5$ $\mathrm{Hz}$; (d) $\Delta f=-2.7 \mathrm{~Hz}$. The slow scan direction is from top to bottom [(a) and (d)] and is from bottom to top (b).

force induced by the tip. It has been demonstrated that at close distances, the chemical force-field associated to the tip-surface interaction induces significant tip-induced local reduction of energy barriers, ${ }^{32,33}$ and the adsorbate experiences an attractive force toward tip. 


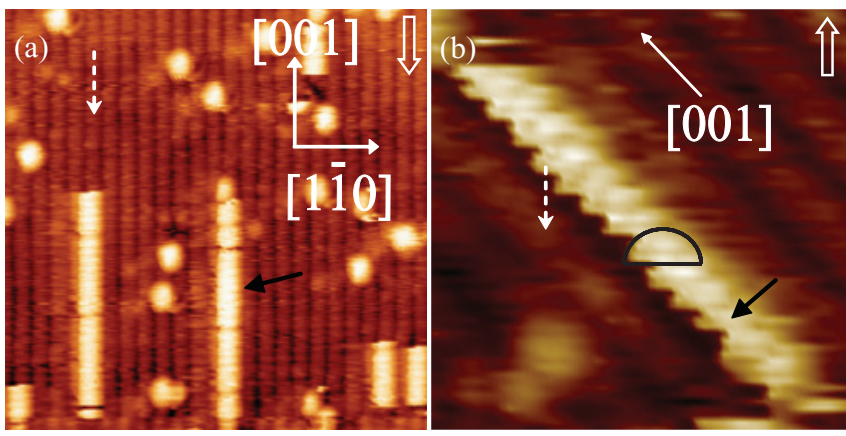

FIG. 5. (Color online) nc-AFM images of the $\mathrm{TiO}_{2}(110)$ surface with relatively higher $\mathrm{K}$ coverage, which illustrate the mobility of $\mathrm{K}$ atoms observed with a tip generating hidden mode image pattern at $80 \mathrm{~K}$. Tip-induced single $\mathrm{K}$ atom manipulation along the path (a) 0 , and (b) 45 degree with respect to the slow-scan direction. The $\mathrm{K}$ atoms (marked by black arrows) preferably migrate along the $\mathrm{O}_{\mathrm{br}}$ atomic rows (dashed white arrows). From the shape and orientation of halfmoon with respect to slow-scan direction, we identify the mechanism of manipulation as pulling mode. The acquisition parameters were [(a) and (b)] $f_{0}=164.398 \mathrm{kHz}, k=51.9 \mathrm{~N} / \mathrm{m}, \Delta f=-3.5 \mathrm{~Hz}$, $U_{\text {cpd }}=1.0 \mathrm{~V}$, and $A=11.5 \mathrm{~nm}$. Image size: (a) $16 \times 16 \mathrm{~nm}^{2}$; (b) $4 \times 4 \mathrm{~nm}^{2}$. The slow scan direction is indicated by the arrows in the upper right corner in each image.

A series of schematic illustrations shown in Fig. 4(e) explain the tip-induced K-atom motion that gives rise to the apparent bright atomic rows in Figs. 4(a), 4(b), 5(a), and 5(b). First, the tip scans the surface from left to right in the fast scan direction and then laterally approaches the $\mathrm{K}$ atom in the slow scan direction. As a result, the $\mathrm{K}$ atom is attracted along the [001] direction toward the tip. As the tip crosses over the top of $\mathrm{K}$ atom along the [1 $\overline{1} 0$ ] direction [step (1) in Fig. 4(e)], no movement of the $\mathrm{K}$ atoms is observed due to the symmetry of the force field. In the subsequent scans, the $\mathrm{K}$ atom is repeatedly imaged as small protrusions until the lower edge of the atom [steps (2) and (3)]. Finally, the $\mathrm{K}$ atom is pulled and jumped in the slow scanning direction due to the influence of an attractive force exerted by the tip [configuration (4)]. This process is repeated by following sequences of imaging and manipulation of $\mathrm{K}$ atom along the $\mathrm{O}_{\mathrm{br}}$ rows. The observed shape of the $\mathrm{K}$ atom with respect to each scan position is indicated in the lower panel of Fig. 4(e).

Figures 4(b) and 4(d) are consecutively recorded nc-AFM images obtained with different imaging set points (i.e., tipsample distances). It is clearly evident that the fluctuations of the apparent positions of $\mathrm{K}$ atoms along the [110] and [001] directions at a close tip-sample distance [Fig. 4(b)] are suppressed at a larger tip-sample distance (smaller tip-K atom interaction) [Fig. 4(d)]. Although both the thermal activation and the tip-induced effects play a significant role for the observed $\mathrm{K}$-atom motion, it is the tip-adatom interaction that has the decisive role. This manipulation process can be facilitated by the tip through a local modification of the surface energy barrier near the limit that enables a thermal hopping. ${ }^{34}$ Moreover, the consecutive nc-AFM images shown in Figs. 4(a) and 4(b) provide additional information on the influence of the surface impurity defects such as $\mathrm{OH}$ groups on the manipulation pathways of adsorbates. Comparison of these images reveals that the effect of the neighboring $\mathrm{H}$ atoms in the cross-row manipulation of $\mathrm{K}$ atoms is evident- the $\mathrm{K}$-atom adsorbate prefers the $\mathrm{OH}$-free path for diffusion across the $\mathrm{Ti}_{5 f}$ rows.

To analyze the influence of the tip-apex termination on $\mathrm{K}$-atom manipulation procedure, we have performed a K-atom manipulation experiment with a distinct type of tip-generating hidden mode image contrast of the $\mathrm{TiO}_{2}$ surface as shown in Figs. 5(a) and 5(b). Here the bright protruding atomic rows were assigned to $\mathrm{O}_{\mathrm{br}}$ atoms and are the favorable channel for $\mathrm{K}$-atom motion. Although the chemical identity of the tip-apex is changed as compared with the tip composing the images in Fig. 4, interestingly, the manipulation of the $\mathrm{K}$ atoms is also achieved with an attractive tip-surface interaction force, i.e., pulling mode. The independence of the manipulation modes from the tip termination, as well as the protruding appearances of the $\mathrm{K}$ atoms in different contrast modes, suggest that the interaction between the tip-apex and $\mathrm{K}$ atoms is attractive in both cases.

\section{Diffusion pathways of $\mathrm{K}$ atoms on $\mathrm{TiO}_{2}(110)$ surface}

In order to identify the most energetically favorable channels and underlying mechanisms for the diffusion of $\mathrm{K}$ atoms on the $\mathrm{TiO}_{2}(110)$ surface, the energy barriers involved in the different hopping paths were computed using first-principles DFT calculations. The energy barriers along selected diffusion paths were simulated using the NEB method ${ }^{23}$ for a $1 \times 2$ surface-unit model. Although the surface impurity defects, i.e., $\mathrm{OH}$ groups are situated in the $\mathrm{O}_{\text {br }}$ rows of $\mathrm{TiO}_{2}$ surface, the $\mathrm{K}$ surface diffusion mostly occurred in the nondefective (OH-free) surface area [see Fig. 4(a)]. Therefore, the pristine surface model without $\mathrm{OH}$ groups was employed for the purpose of revealing the pathways and energetics of the $\mathrm{K}$ diffusion on the defect-free surface area.

The diffusion-energy profile, calculated as a function of the normalized migration path, is shown in Fig. 6(a) for various pathways, as depicted in Fig. 6(b): ( $3 \rightarrow 4 \rightarrow 3,3 \rightarrow 6 \rightarrow$ $3,3 \rightarrow 4 \rightarrow 5 \rightarrow 4 \rightarrow 3$, and $6 \rightarrow 1 \rightarrow 6$ ). Two different pathways, $3 \rightarrow 4 \rightarrow 3$ and $3 \rightarrow 6 \rightarrow 3$, were identified as the most energetically favorable channels for the diffusion of a $\mathrm{K}$ atom located on the upper hollow site toward the adjacent site, which is consistent with the experimental results in terms of the manipulation pathways. At farther tip-sample distances, as in Fig. 2(c), switching of the K-atom position across the $\mathrm{O}_{\mathrm{br}}$ rows is observed, which is evident of a small barrier for atomic motion along this direction. At closer distances, the $\mathrm{K}$ atoms begin to move along the $\mathrm{O}_{\text {br }}$ rows [Figs. 4(a), 4(b), 5(a), and 5(b)]. In accordance, DFT calculations reveal a low-energy barrier of $0.16 \mathrm{eV}$ for cross-row diffusion between two adjacent hollow sites $(3 \rightarrow 4 \rightarrow 3)$ via the bridge site (site 4), which was considered a transition state of $\mathrm{K}$ as it diffuses across the $\mathrm{O}_{\mathrm{br}}$ rows. On the other hand, a higher-energy barrier is found for the path $(3 \rightarrow 4 \rightarrow 5 \rightarrow 4 \rightarrow 3)$ over the $\mathrm{O}_{\mathrm{br}}$ row. Since the center of the moving $\mathrm{K}$ atoms is located on the $\mathrm{O}_{\mathrm{br}}$ row, and is extended between two $\mathrm{Ti}_{5 f}$ rows, the low-energy barrier across the $\mathrm{O}_{\mathrm{br}}$ row suggests the observed extended shape of $\mathrm{K}$-atom protrusion around $\mathrm{O}_{\mathrm{br}}$ rows is due to the rapid movement of $\mathrm{K}$ atoms across the $\mathrm{O}_{\mathrm{br}}$ row compared with the acquisition speed of AFM 

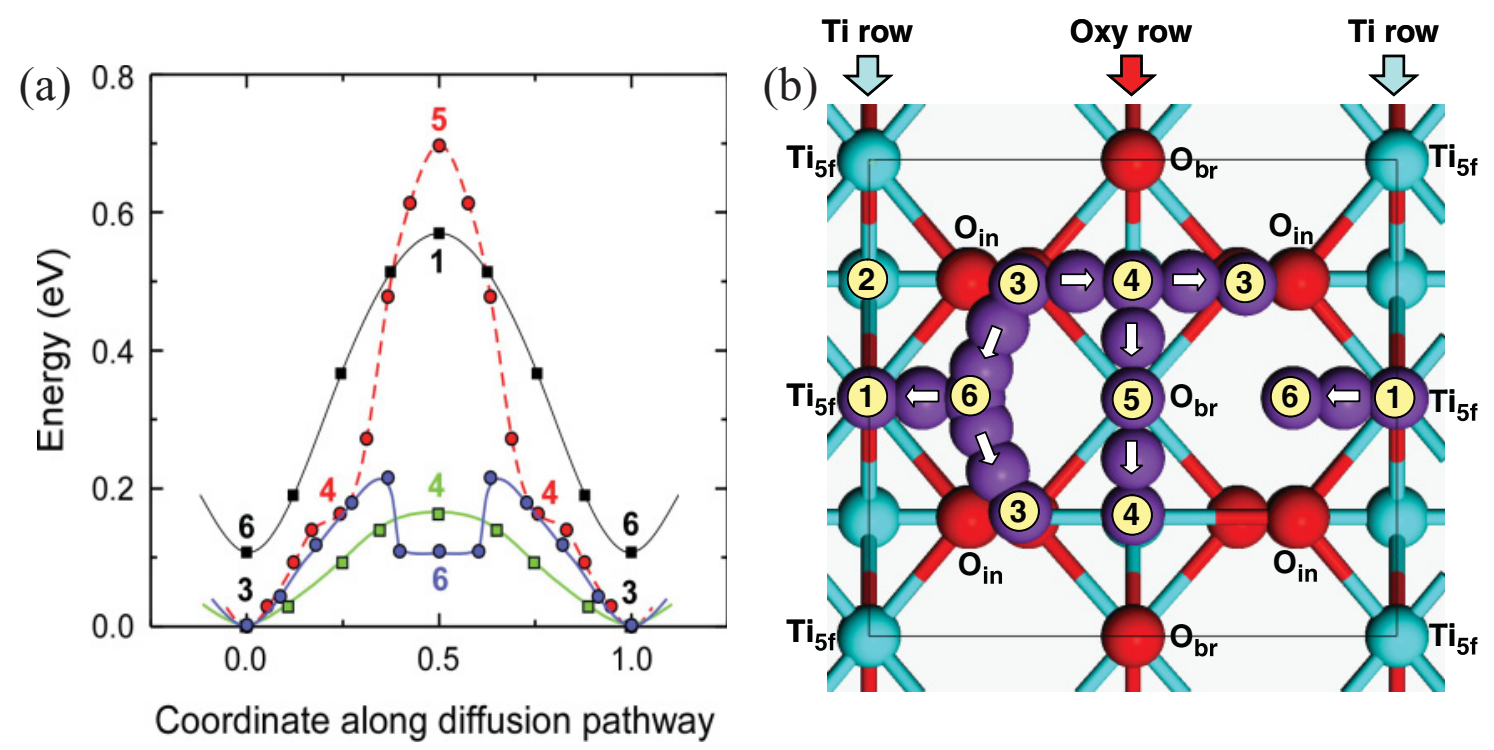

FIG. 6. (Color online) (a) Calculated energy landscape for K-atom diffusion along different pathways on the $\mathrm{TiO}_{2}(110)$ surface for a $(1 \times 2)$ model. (b) Ball model of the $\mathrm{TiO}_{2}(110)$ surface with a $\mathrm{K}$ atom adsorbed on different sites, indicating the possible diffusion paths. The sites considered in plotting the diffusion profiles are on the top of $\mathrm{Ti}_{5 f}$ atoms (1), between two $\mathrm{Ti}_{5 f}$ atoms (2), between two $\mathrm{O}_{\mathrm{br}}$ atoms and one $\mathrm{O}_{\text {in }}$ atom on a basal plane (3), between two $\mathrm{O}_{\mathrm{br}}$ atoms (4), on the top of an $\mathrm{O}_{\mathrm{br}}$ atom (5), and between two $\mathrm{O}_{\text {in }}$ atoms and one $\mathrm{O}_{\mathrm{br}}$ atom (6). The energies are estimated relative to the K-atom configuration at the upper hollow site (i.e., site 3). The points correspond to actual calculations that were fitted to a smooth curve. The arrows indicate the diffusion path directions considered for the DFT calculations of (a). The horizontal axis of the curves in (a) is defined as the ratio of the migration distance (from the starting to a point on the path) to total distance between start and end points.

(see Figs. 4 and 5). The time average of this process was experimentally observed; therefore, the diffusion along the $\mathrm{O}_{\mathrm{br}}$ row is indeed accomplished via sites 3, 4, and 6, which are on either side of an $\mathrm{O}_{\mathrm{br}}$ row. Along this low-energy channel, the $\mathrm{K}$ atoms tend to keep two $\mathrm{O}_{\mathrm{br}}$ and one $\mathrm{O}_{\text {in }}$ atom (or vice versa) neighbors to maximize the contact with surface oxygen and preserve a strong $\mathrm{K}-\mathrm{O}$ ionic bond along this diffusion path. However, along the $3 \rightarrow 6 \rightarrow 3$ path, an additional $\mathrm{K}$ atom bonds weakly with a $\mathrm{Ti}_{5 f}$ atom, which results in a small local minimum at site 6 . The interaction around site 6 may involve a mixture of ionic interaction between $\mathrm{K}^{\delta+}$ and $\mathrm{O}^{\alpha-}$ atoms and orbital hybridization of the $\mathrm{K} 4 s$ and $\mathrm{Ti}_{5 f} 3 d$ states.

Although, statistically, the majority of the $\mathrm{K}$ atoms are manipulated along the [001] direction, cross-row displacements of $\mathrm{K}$ atoms are also observed in Figs. 4(a) and 4(b). In contrast to nc-AFM images showing cross-row displacement of $\mathrm{K}$ atoms, a large energy barrier of $0.58 \mathrm{eV}$ across $\mathrm{Ti}_{5 f}$ atomic rows $(6 \rightarrow 1 \rightarrow 6)$ was determined from first-principles calculations. However, the presence of the tip-induced effects, which have an influence on the localized barrier reduction near the limit that allows thermally activated motion at $80 \mathrm{~K}$, was not included in our DFT calculations. In addition, it has been shown that the diffusion barrier depends on the local adjacency of neighboring adsorbed atoms, ${ }^{35}$ because adsorbate-adsorbate interactions drastically modify the potential energy landscape of an adsorbed atom on the surface. Therefore, the consideration of these effects will facilitate overcoming the high-energy barrier for the $\mathrm{K}$-atom motion across the $\mathrm{Ti}_{5 f}$ rows along the [1 10$]$ direction.

\section{CONCLUSION}

We have investigated the adsorption and tip-induced manipulation of $\mathrm{K}$ atoms on $\mathrm{TiO}_{2}(110)$ surfaces at $80 \mathrm{~K}$ through a combined nc-AFM and DFT study. At submonolayer coverage, first-principles GGA $+U$ calculations reveal favorable adsorption sites that are consistent with the experimentally observed adsorption positions on the upper threefold hollow site. We have shown that the manipulation of adsorbed $\mathrm{K}$ atoms, which occurs mainly along the $\mathrm{O}_{\mathrm{br}}$ rows, can be achieved by the tip through attractive force, i.e., pulling mode. The determined diffusion-energy barriers for a single $\mathrm{K}$ atom along different pathways show good agreement with the experimentally obtained manipulation paths. The low-energy path goes from one upper hollow site to another either through bridge or lower hollow sites, with an associated diffusionenergy barrier of 0.16 and $0.21 \mathrm{eV}$, respectively. This study is an important step toward understanding the mechanisms and pathways to perform manipulation of adsorbed metal atoms on metal oxides and serves as the basis for further investigation of the dependence of catalytic performance on particle size.

\section{ACKNOWLEDGMENTS}

This work was supported by Grants-in-Aid for Scientific Research (22221006, 19053006, 21246010, 21656013, and 22760028) from the Ministry of Education, Culture, Sports, Science and Technology (MEXT) of Japan, Funding Program for Next Generation World-Leading Researchers, the Japan Science and Technology Agency (JST), Handai FRC, the Atomic Technology project funded by MEXT, and Global COE programs. 
*ayhan@afm.eei.eng.osaka-u.ac.jp

${ }^{1}$ U. Diebold, Surf. Sci. Rep. 48, 53 (2003).

${ }^{2}$ V. E. Henrich and P. A. Cox (eds.), The Surface Science of Metal Oxides (Cambridge University Press, Cambridge, UK, 1994).

${ }^{3}$ R. Heise and R. Courths, Surf. Sci. 331, 1460 (1995).

${ }^{4}$ A. W. Grant and C. T. Campbell, Phys. Rev. B 55, 1844 (1997).

${ }^{5}$ A. Sasahara, H. Uetsuka, and H. Onishi, Jpn. J. Appl. Phys. 43, 4647 (2004).

${ }^{6}$ M. Valden, X. Lai, and D. W. Goodman, Science 281, 1647 (1998). ${ }^{7}$ R. Souda et al., Surf. Sci. 285, 265 (1993).

${ }^{8}$ A. M. Kiss, M. Svec, and A. Berko, Surf. Sci. 600, 3352 (2006).

${ }^{9}$ S. Fernandez, A. Markovits, and C. Minot, J. Phys. Chem. C 112, 14010 (2008).

${ }^{10}$ N. Oyabu, O. Custance, I. Yi, Y. Sugawara, and S. Morita, Phys. Rev. Lett. 90, 176102 (2003).

${ }^{11}$ Y. Sugimoto et al., Nat. Mater. 4, 156 (2005).

${ }^{12}$ M. Ternes et al., Science 319, 1066 (2008).

${ }^{13}$ S. Hirth, F. Ostendorf, and M. Reichling, Nanotechnology 17, S148 (2006).

${ }^{14}$ F. Loske and A. Kühnle, Appl. Phys. Lett. 95, 043110 (2009).

${ }^{15}$ N. Suehira et al., Rev. Sci. Instrum. 72, 2971 (2001).

${ }^{16}$ T. R. Albrecht et al., J. Appl. Phys. 69, 668 (1991).

${ }^{17}$ S. Wendt et al., Surf. Sci. 598, 226 (2005).

${ }^{18}$ S. L. Dudarev, G. A. Botton, S. Y. Savrasov, C. J. Humphreys, and A. P. Sutton, Phys. Rev. B 57, 1505 (1998).

${ }^{19}$ G. Kresse and D. Joubert, Phys. Rev. B 59, 1758 (1999).
${ }^{20}$ P. E. Blöchl, Phys. Rev. B 50, 17953 (1994).

${ }^{21}$ K. Yang, Y. Dai, B. Huang, and Y. P. Feng, Phys. Rev. B 81, 033202 (2010).

${ }^{22}$ H. J. Monkhorst and J. D. Pack, Phys. Rev. B 13, 5188 (1976).

${ }^{23}$ G. Mills and H. Jönsson, Phys. Rev. Lett. 72, 1124 (1994).

${ }^{24}$ U. Diebold, J. F. Anderson, K. O. Ng, and D. Vanderbilt, Phys. Rev. Lett. 77, 1322 (1996).

${ }^{25}$ K. I. Fukui, H. Onishi, and Y. Iwasawa, Phys. Rev. Lett. 79, 4202 (1997).

${ }^{26}$ G. H. Enevoldsen, H. P. Pinto, A. S. Foster, M. C. R. Jensen, A. Kuhnle, M. Reichling, W. A. Hofer, J. V. Lauritsen, and F. Besenbacher, Phys. Rev. B 78, 045416 (2008).

${ }^{27}$ A. Yurtsever et al., Nanotechnology 21, 165702 (2010).

${ }^{28}$ C. L. Pang et al., Surf. Sci. 583, L147 (2005).

${ }^{29} \mathrm{~A}$. Yurtsever et al. (in preparation).

${ }^{30}$ J. T. Li, W. D. Schneider, and R. Berndt, Appl. Phys. A 66, S675 (1998).

${ }^{31}$ L. Bartels, G. Meyer, and K.-H. Rieder, Phys. Rev. Lett. 79, 697 (1997).

${ }^{32}$ Y. Sugimoto, P. Jelinek, P. Pou, M. Abe, S. Morita, R. Perez, and O. Custance, Phys. Rev. Lett. 98, 106104 (2007).

${ }^{33}$ M. B. Watkins and A. L. Shluger, Phys. Rev. B 73, 245435 (2006).

${ }^{34}$ M. Watkins, T. Trevethan, A. L. Shluger, and L. N. Kantorovich, Phys. Rev. B 76, 245421 (2007).

${ }^{35}$ A. Taranovskyy, T. Tansel, and O. M. Magnussen, Phys. Rev. Lett. 104, 106101 (2010). 